\title{
«Bilbao Dendak»: una experiencia de partenariado público-privado para la promoción comercial de la ciudad de Bilbao
}

\section{Jon Aldeiturriaga Barrenechea *}

\section{Breves apuntes históricos}

La tradición comercial de Bilbao, origen de la Villa, es una constante de la historia que nos remonta al primer asentamiento de marinos y mercaderes de comienzos del siglo XIV, al comercio de las naves vizcaínas, con los puertos de Burdeos, Sevilla, Brujas, etc., del siglo XVI, o la expansión comercial del xVIII.

El 15 de junio del año 1300, Don Diego López de Haro, Señor de Vizcaya, firma en Valladolid la carta puebla que transforma en Villa al pequeño pueblo de pescadores y mercaderes situado a orillas de la Ría del Nervión y ubicado hacia la zona donde hoy se encuentra la iglesia de San Antón.

La carta puebla fundacional de la Villa de Bilbao otorga a ésta el monopolio del tráfico por la ría y el de numerosas transacciones mercantiles en una amplia área geográfica.

A lo largo del siglo XIv se desarrolla una importante actividad comercial reforzada con la creación de astilleros y ferrerías como complemento de las explotaciones mineras existentes en la zona.

La actividad comercial de sus calles es acorde con el desarrollo mercantil cara al exterior, de forma que para comienzos del siglo xvi Bilbao se habrá convertido en el principal puerto del País Vasco. En 1511 se crea el denominado «Consulado, Casa de Contratación, Juzgado de los hombres de negocios de mar y tierra» conocido como «Consulado de Bilbao», que será el gran dinamizador de la vida económica. En este tiempo se desarrolla un intenso tráfico comercial con Brujas, Nantes y se entra en la carrera de las Indias. El Consulado de Bilbao llegó a ser el auténtico gobierno de la Villa, ya que los comerciantes que pertenecían al mismo estaban facultados para nombrar Alcalde y otras autoridades entre las que se encontraba el importantísimo Cónsul, responsable del tráfico por la Ría.

Bilbao, que no ha dejado de crecer económicamente desde su fundación, experimentará un declive a finales del siglo Xvi. El Comercio empieza a perder importancia debido, entre otras causas, a la disminución de la demanda europea del hierro vasco y a los numerosos conflictos bélicos mantenidos por la casa de Austria.

Desde 1600 , se inicia una nueva estrategia comercial que tendrá efectos espléndidos, convirtiéndose Bilbao en el centro de contratación de los mercaderes europeos de la lana. El siglo XVIII supone para Bilbao la irrupción del capitalismo comercial. A través del Consulado de Bilbao se promoverán las ordenanzas de 1737, código comercial considerado ejemplar, adoptado en España y seguido por numerosos países.

El Plan Loredo de 1785 planta las raíces de lo que sería el Bilbao del futuro, ya que al entrar en el siglo xix, Bilbao empezará a sentir el agobio urbanístico consecuencia de las 
posibilidades límite de crecimiento espacial, lo que hará que se empiece a pensar en ensanches de futuro al otro lado de la ría. Este nuevo Bilbao se incorporará rápidamente a la actividad comercial y de servicios, destacando entre éstos la actividad bancaria y bursátil, sin olvidarnos de la Feria Internacional de Muestras.

\section{El asociacionismo comercial en Bilbao}

La historia del asociacionismo comercial en Bilbao comienza con el nacimiento de la Asociación de Comerciantes del Casco Viejo en 1969, si bien diez años antes se comienzan a dar los primeros pasos entre comerciantes de algunas calles, que se traducen en sorteos y promociones conjuntas y en acciones de urbanismo como la peatonalización de la calle Carnicería Vieja. Todas estas acciones tienen lugar en el ámbito del Centro Histórico de Bilbao.

En estos momentos existen en la Villa diez asociaciones de comerciantes de carácter espacial. Estas asociaciones actúan tanto sobre el centro de la ciudad como sobre los diferentes barrios de Bilbao. Aglutinan en total a más de 1.400 comercios.

En orden a su antigüedad son las siguientes:

- ASOCIACIÓN DE COMERCIANTES DEL CASCO VIEJO. Formada por 300 comerciantes y hosteleros, engloba al 50\% de los empresarios del Centro Histórico. Nace en 1969 y su objetivo fundamental es la promoción comercial del Casco Viejo, atrayendo el mayor número de visitantes, potenciales compradores, a la zona, no sólo mediante acciones promocionales y publicitarias, sino potenciando también actividades de tipo socio-cultural y urbanístico de la Villa que redunda en evidente beneficio de los comerciantes aquí instalados. Especial atención dedica a la mejora de la capacitación profesional de comerciantes y trabajadores del comercio, mediante la formación continua y el reciclaje. Cuenta con un equipo profesional de cuatro personas. En sus locales se ubica uno de los Gabinetes de Asistencia Técnica al Comercio - GATC - puestos por el Gobierno autonómico a disposición de los comerciantes bilbaínos.

- AGRUPACIÓN DE COMERCIANTES DE SANTU. IXU. Con 250 empresarios asociados, nace en 1976 en uno de los barrios más populosos de la Villa y con una fuerte personalidad. Sus objetivos se cifran en la defensa de los intereses generados en el sector comercial y la representación de los mismos, con el objetivo último de evolucionar hacia un modelo eficaz de comercio profesionalizado, asociado y especializado.
Cuenta con un administrativo a su servicio así como los servicios de otro GATC, que atiende también a las asociaciones de Txurdinaga y a la de Otxarkoaga.

- ASOCIACIÓN DE COMERCIANTES DEL MERCA. DO DE LA RIBERA. Nace en 1977 y cuenta en la actualidad con 140 asociados. Esta Asociación de Comerciantes del Mercado cubierto más grande de Europa - Guiness record - tiene en su haber la más alta representatividad, pues representa a más del $75 \%$ de los asentadores. Se encuentra en proceso de selección para la contratación de un gerente profesional. Los mismos comerciantes han formado una Sociedad Mercantil -MERCADO DE LA RIBERA, S. A.- que debate con el Ayuntamiento -el mercado es municipal - la autogestión y una profunda reforma en la que los comerciantes podrían invertir más de $500 \mathrm{Mptas}$. Sus objetivos estatutarios son la representación, gestión y promoción de los intereses económicos, profesionales, sociales y culturales de los afiliados.

- ASOCIACIÓN DE COMERCIANTES DE SAN FRANCISCO. Se constituye en 1983 y está formada en la actualidad por más de 50 comerciantes. Regida por una Junta Directiva de carácter altruista, no cuenta con personal liberado en la actualidad. Sus objetivos son: dinamizar el comercio en su ámbito de actuación y colaborar en la regeneración socio-urbanística y humana de Bilbao la Vieja, donde se ubica, una de las zonas más degradadas del Estado.

- ASOCIACIÓN DE COMERCIANTES DE DEUSTO Y SAN IGNACIO. Fundada en 1986, reúne a 60 comerciantes cuyo objetivo pasa por mejorar sus estructuras y fomentar la atención al cliente. Durante este año va a realizar una campaña de captación de asociados que le permita mejorar su representatividad, así como culminar la preparación de sus asociados ante la inminente llegada del Euro.

- AGRUPACIÓN DE COMERCIANTES DEL CEN. TRO COMERCIAL BIDARTE. 45 comerciantes forman la Asociación de esta galería comercial ubicada en el barrio de Deusto, de fuerte personalidad y que permaneció independiente de Bilbao hasta los años veinte del pasado siglo. Barrio universitario por excelencia, ya que en él se encuentra la conocida Universidad de Deusto. La Asociación nace en 1994 y tiene como objetivo la defensa ordenada y legal de los intereses de sus asociados, fomentando la colaboración entre todos los comerciantes y promocionando el Centro Comercial Bidarte, cuya gerencia, desempeñada por Jones Lang LaSalle, administra la Asociación con un equipo habitualmente compuesto por 2 03 personas.

- AGRUPACIÓN CENTRO COMERCIAL INDAUTXU. Asociación nacida en 1995, cuenta en la actualidad con 225 socios, siendo su objetivo primordial la promoción y difusión de la oferta comercial, para aumentar la capacidad competitiva y el atractivo del conjunto del comercio y servicios esta- 
blecidos en Indautxu (Zona Centro de Bilbao), a través de campañas de animación y difusión comercial, dirigidas a mantener y captar nuevos clientes para el comercio de Indautxu. $\mathrm{Al}$ mismo tiempo promover actividades internas dirigidas a la mejora de la información, formación, servicios, modernización de los negocios, etc. Cuenta con equipo gerencial formado por dos personas, una de ellas con carácter ejecutivo.

- TXURDINAGA ASOCIACIÓN DE COMERCIANTES. Con 95 socios, esta joven Asociación de Comerciantes, nacida en 1998, actúa en uno de los barrios de clases medias con un equipamiento comercial más bajo, pero el índice de zonas verdes más amplio. Sus objetivos se centran en activar comercialmente el barrio, fomentar la unión entre comerciantes y su puesta al día, poder acceder a ayudas institucionales para formación, etc., conseguir un GATC propio, así como un local para desarrollar sus actividades.

- GRUPO COMERCIAL ENSANCHE. Nacida en diciembre de 1998, la forman en la actualidad 150 socios. Cuenta con un equipo profesional compuesto por una Gerente y una administrativa; actúa sobre el denominado Ensanche de Bilbao, área urbana delimitada por las plazas Moyúa y Circular y con su eje central en la Gran Vía de Don Diego López de Haro. Sus objetivos se enmarcan en cuatro líneas estratégicas: a) Mejora del marco urbanístico, b) Imagen conjunta del Ensanche bilbaíno, c) Incremento de la competitividad empresarial y d) Animación y promoción del Ensanche. Su Junta Directiva está formada por 17 miembros y tiene comisiones específicas de Comunicación, Servicios a asociados, Urbanismo y de Comités Sectoriales.

- ASOCIACIÓN DE COMERCIANTES DE OTXARKOAGA. Esta Asociación, nacida en julio de 1999, de la mano de la Agencia de Desarrollo Local y Empleo LANEKINTZA, cuenta en la actualidad con 95 socios que trabajan unidos para dar a conocer a la asociación en su ámbito de actuación. Su objetivo es conseguir mejorar y hacer más atractiva la compra a sus clientes, mediante la puesta en marcha de campañas y la presencia institucional. Participan intensamente en la organización de las fiestas locales de su barrio como nexo de unión con sus clientes potenciales.

\section{Antecedentes de Bilbao Dendak}

\section{El cluster de turismo}

A principio de 1995, un reducido grupo de comerciantes y hosteleros comienzan a plantearse la necesidad de dinamizar
Bilbao desde el punto de vista de sus respectivos sectores. Ante las cercanas Elecciones Municipales, deciden consolidar su grupo, ampliando su base, con participación de OPC's, hoteles y empresarios de los sectores turístico y comercial.

Celebradas las Elecciones, y ante la inexistencia de una concejalía de comercio en el gobierno municipal, se plantea la idea en el Área Municipal de Cultura y Turismo, por entender que la dinamización del comercio genera e incrementa el atractivo de una ciudad.

El proyecto define por primera vez en el País Vasco el concepto de competencia entre «ciudad y periferia», presentado en el Congreso de la Asociación Española de Centros Comerciales celebrado en Barcelona por la Asociación de Comerciantes del Casco Viejo, abandonando el antiguo concepto de «grandes contra pequeños» que se venía manejando hasta la fecha.

En esta época se comienzan a aplicar en el País Vasco las teorías de Michael Porter y en el mes de julio, a efectos de valorar la necesidad de poner en marcha de forma definitiva el proyecto, se constituye un grupo estable de trabajo al que, además de comerciantes, hosteleros y OPC's, se unen hoteles, Agencias de viaje, la Cámara de Comercio, la Feria Internacional de Muestras y la Oficina de Turismo como coordinadora de los trabajos.

Este grupo trabaja con un alto nivel de motivación al entender imprescindible el diseño de una política turístico-comercial consensuada y la coordinación de acciones que, planificadas conjuntamente entre la iniciativa pública y privada, pueden alcanzar mayor rendimiento para la ciudad al mismo costo. El grupo era conocido como EL CLUSTER DE TURISMO.

Fruto de estos trabajos y del apoyo municipal decidido, surge la «Iniciativa de Refuerzo de la Competitividad en Turismo de Bilbao», estudio que intenta motivar al cambio positivo a los diferentes agentes relacionados con el sector. Se divide en tres fases:

1. Lanzamiento de la iniciativa.

2. Consensuar una dirección estratégica amplia para la ciudad.

3. Definir líneas de actuación y estructurar los mecanismos que sirvan para iniciar el cambio.

Fruto de esta experiencia, en la que participan más de cien personas de otras tantas Entidades y empresas, surgen una serie de proyectos definidos como unidades de negocio:

A. Casco Viejo, Centro Comercial, Turístico, Monumental y Gastronómico.

B. Ensanche, Moda y Arte.

C. Bilbao Cultural.

D. Bilbao, Centro de Congresos y Convenciones. 


\section{Secores implicados por la inicintiva}

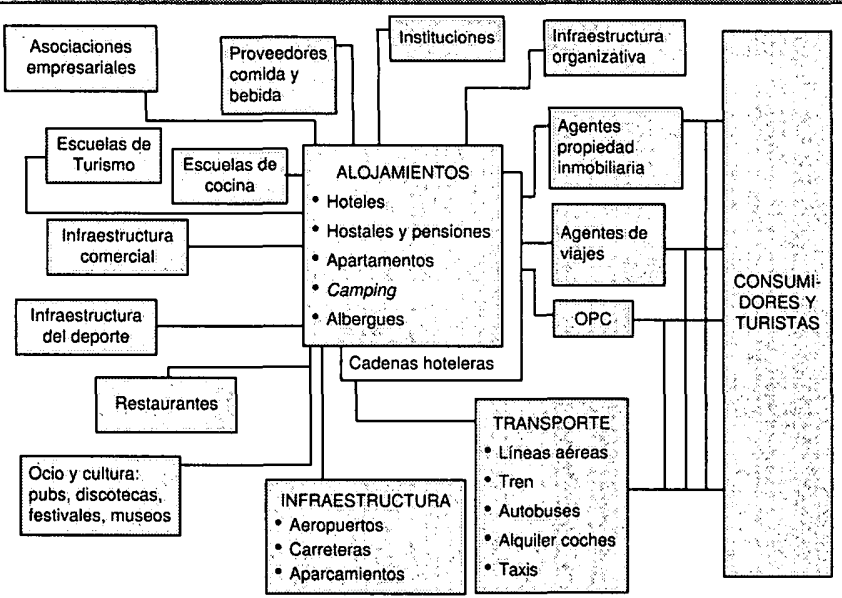

Con el paso del tiempo, a iniciativa de la Cámara de Comercio, y con el fin de generar sinergias, con la base de los grupos Casco Viejo y Ensanche se crea el grupo «Mesa de Comercio de Bilbao», embrión de Bilbao Dendak.

\section{La Mesa de Comercio de Bilbao}

La Mesa de Comercio inicia sus trabajos en base a las conclusiones emanadas del informe final del Plan de Refuerzo de la Competitividad Turística de Bilbao, complementadas con las de: la Declaración de Málaga, actuando en los siguientes capítulos:

\section{Relación con las Administraciones}

1. Presentación del Plan Bienal de actuación al equipo municipal recientemente constituido para tratar de sensibilizar y conseguir el compromiso de responsables políticos y técnicos de las áreas municipales competentes en las diferentes materias contempladas en el Plan.

2. Solicitar del Gobierno Vasco la realización de un estudio complementario al fallido PERCO del año 1995 que permita una aproximación más veraz a la realidad comercial de Bilbao. Dicha solicitud la realizará el Ayuntamiento de Bilbao en nombre y representación de los miembros de la Mesa de Comercio.

3. Solicitar del Ayuntamiento de Bilbao cauces para establecer una relación adecuada entre las Asociaciones de Comerciantes y los diferentes Consejos de Distrito, a fin de canalizar las necesidades zonales.

Se trata fundamentalmente de reforzar la relación natural entre el comercio y la renovación urbana, para poder abordar aspectos como:
- Limpieza.

- Jardinería.

- Iluminación.

- Fachadas.

- Mobiliario urbano.

- Peatonalización.

- Aparcamientos.

A tal efecto se propone que cada zona de Bilbao elabore un plan de necesidades que pueda servir de documento de trabajo.

En este capítulo el cometido de la Mesa de Comercio es el de realizar gestiones oportunas para conseguir la presencia necesaria y suficiente de las Asociaciones en dichos Consejos de Distrito, puesto que en ellos se priorizan diferentes inversiones en infraestructuras que afectan al comercio.

4. Se valora la conveniencia de invitar al Gobierno Vasco a formar parte del Consejo Director de la Mesa de Comercio.

\section{Identificación}

5. Definición de las zonas comerciales de Bilbao, creando o potenciando focos de centralidad y estableciendo jerarquías de centros, según especialización.

6. Realización de un estudio global de señalización con diseño propio de Bilbao.

7. Creación de nombre y logotipo.

\section{Calidad}

8. Reciclaje y puesta al día de los trabajadores del comercio, siendo el cometido de la Mesa el de estudiar las necesidades de formación prioritarias, contactando con aquellas Instituciones capaces de satisfacer las necesidades en este sentido.

9. Definición de normas de calidad para el comercio de Bilbao, profesionalizándose este aspecto a través de un estudio que puede derivarse del PERCO, o bien encargarse a una consultora especializada en este tema.

10. Impulso al asociacionismo, a fin de que la interlocución de las asociaciones existentes sea más representativa, se encuentren soportadas económicamente por mayor número de establecimientos y los acuerdos sean vinculantes.

\section{Ocio y comercio}

11. Articulación de propuestas de ocio y comercio que refuercen ambos sectores, trabajando en un plan que refleje: 
a) Itinerarios mixtos.

b) Acciones de dinamización.

c) Estudio comparativo de ciudades en relación a apertura en festivos y horario comerciales.

12. Continuar con la campaña de Navidad de promoción de Bilbao como ciudad de compras

\section{Consideraciones finales}

Este plan nace con un plazo de ejecución bienal (1999-2000), sin referencias a acciones concretas, en tanto en cuanto las negociaciones políticas y la disponibilidad económica van a afectar de forma determinante al mismo.

Sin embargo, el proceso se acelera debido al dinamismo de los participantes y al decidido apoyo de la Administración Municipal, por lo que ya en el mes de mayo del año 2000 nace.

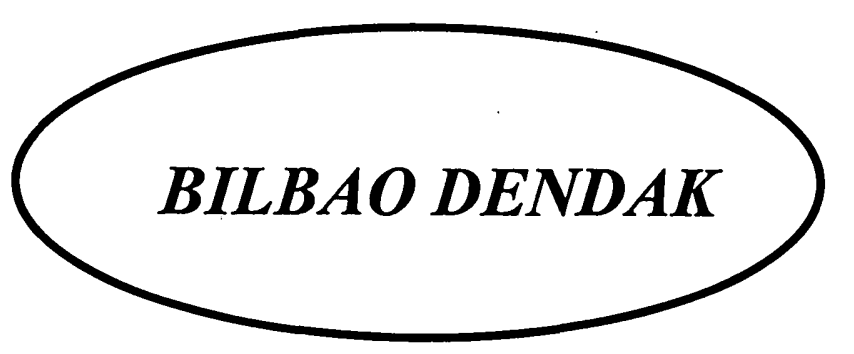

\section{Bilbao Dendak (Tiendas de Bilbao)}

\section{El proyecto}

\section{Introducción}

El objetivo de este proyecto es el estudio de puesta en marcha de acciones dinamizadoras del comercio bilbaíno.

Las funciones que debiera cumplir son:

- Aceptar los retos necesarios para contribuir a la modernidad de Bilbao.

- Afianzar el liderazgo comercial de Bilbao en el eje Atlántico.

- Dinamizar al comerciante haciéndole trabajar en pos de retos concretos.

- Mejorar el posicionamiento social del comercio y del comerciante.

\section{Propuesta de actividades}

\subsection{Foro Bilbao Dendak}

Foro de debate y análisis del comercio de Bilbao formado por miembros de Bilbao Dendak, así como por representantes institucionales, universidades, expertos, etc... Sus objetivos serán, entre otros:

- Reflexionar sobre el papel del comercio urbano y su futuro.

- Generar Jornadas de Debate sobre diversos temas de interés para el comercio.

- Fomentar la interacción comercio-Universidad.

- Desarrollar un plan de cursos ligado a la Universidad de Verano de la UPV.

- Diseñar una titulación, diplomatura o similar, que permita el acceso o el reciclaje en la profesión en condiciones mejores a las actuales, lo que garantizaría una mayor eficacia y competitividad en el comercio de Bilbao.

- En determinadas facultades se podrían promover estudios y tesis sobre el comercio y su historia, lo que podría dar origen a publicaciones, incluso a un libro sobre el comercio bilbaíno.

\subsection{Promoción del comercio electrónico}

- Estudiar un plan para la presencia del conjunto del comercio bilbaíno en Internet, mediante la creación de páginas Web de las asociaciones y su acceso a través de la página oficial de Bilbao «www.bilbao.net».

- Diseño y puesta en marcha del Centro Comercial Virtual «e-shoppingBilbao». Para ello se podría promover un acuerdo de colaboración con un socio tecnológico, por una parte, y con los socios bilbaínos del proyecto europeo DIGITAL MARKET, el Centro de Investigación de la Diputación, Robotiker y la Asociación para la Revitalización del Bilbao Metropolitano, Bilbao Metrópoli-30.

\subsection{Día del Comercio}

Acto social que busca el reconocimiento del comercio y los comerciantes como colectivo que estructura la vida de la ciudad y aporta calidad de vida a los ciudadanos.

En este acto se podría dar un reconocimiento oficial a los comerciantes que han puesto al día sus negocios, remodelándolos o abriendo nuevos locales; las asociaciones serían las encargadas de las nominaciones.

Puesto que el primer día se celebraría dentro del 700 aniversario de la Villa y hoy nadie duda del papel que jugaron 
los comerciantes en la fundación de Bilbao, proponemos que en el transcurso de los actos se convoque un concurso para la implantación del «Monumento al Comercio, o al Comerciante».

\subsection{Promoción y mantenimiento de Artesanos Comerciantes}

Viejas profesiones desde siempre ligadas al comercio y que hoy se puede pensar en recuperar ligadas al atractivo turístico de Bilbao. Al estilo de los museos etnográficos del norte de Europa se podría diseñar un espacio polivalente donde estos artesanos (boteros, etc.) trabajarían, tendrían aprendices y venderían sus productos.

\subsection{Premio anual de Periodismo al mejor artículo sobre Comercio de Bilbao}

En colaboración con la Asociación de Prensa de Vizcaya, se establecerán las bases de un premio anual al mejor trabajo periodístico que contribuya a difundir y destacar el papel del comercio de Bilbao en nuestra ciudad.

\subsection{Comunicación Global}

- Nombre oficial de la «mesa»: BILBAO DENDAK.

- Diseño y adopción de un logotipo aglutinador de las acciones de la Entidad gestora del proyecto.

- Plan Estratégico de comunicación

- Definir y difundir la imagen del comercio.

- Campañas de promoción.

- Contacto con los medios de comunicación: ruedas de prensa, etc.

- Promover una sección fija sobre el comercio en los diarios bilbaínos y en el periódico municipal Bilbao.

\section{Funcionamiento}

\subsection{Definir tipo de organización}

Dotar a Bilbao Dendak de una forma jurídica, que garantice la participación y representación de todos los agentes institucionales, públicos y privados que conforman la actual mesa de comercio de Bilbao.

Asimismo, la forma jurídica debe ser de tales características que permita agilidad de funcionamiento de las diferentes áreas de actuación, así como el acceso a fórmulas de financiación institucionales y cualquier otro tipo de ayudas. A futuro se estu- diará, con detenimiento, la constitución de una Fundación en torno a Bilbao Dendak.

\subsection{Sistema de Financiación}

Bilbao Dendak deberá diseñar y consensuar un sistema de financiación que compatibilice las aportaciones de los miembros, las ayudas públicas y privadas y los fondos propios que se generen de las actividades que se realicen.

\subsection{Dinámica de trabajo}

Con el fin de llevar adelante la programación de actividades propuesta, se sugiere la siguiente operativa de trabajo:

- El Plenario de Bilbao Dendak se reunirá como mínimo los primeros martes de cada mes, además de todas las veces que se consideren necesarias en función de la dinámica de trabajo.

- Se crearán Comisiones de Trabajo por objetivos fijados por el Plenario de Bilbao Dendak, que se reunirán cuantas veces lo estimen sus miembros.

Para dinamizar este proyecto es imprescindible la existencia de una figura que, con carácter profesional, coordine y ejecute todas las propuestas, velando además por el cumplimiento de los objetivos de Bilbao Dendak: EL GERENTE DE CENTRO URBANO.

\begin{tabular}{|c|c|}
\hline $\mathrm{Am}^{2}+\mathrm{x}$ & 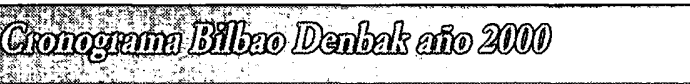 \\
\hline $\begin{array}{l}\text { Mayo: } \\
\text { Junio: }\end{array}$ & 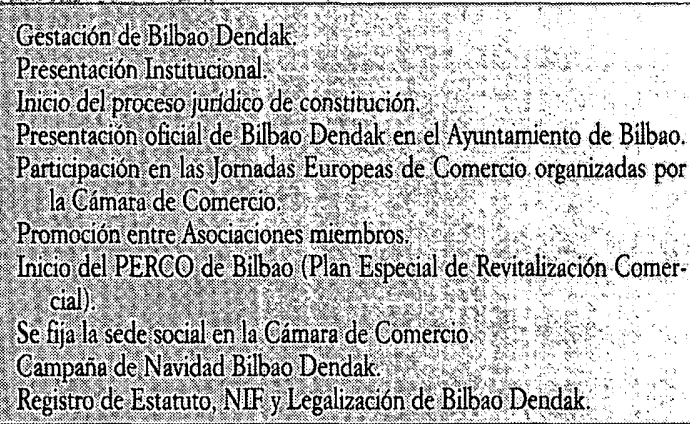 \\
\hline
\end{tabular}

\section{La realidad}

El año 2001 va a ver la consolidación de la organización conocida oficialmente como Asociación para la Revitalización del Sector Comercial de Bilbao «BILBAO DENDAK». La Asociación la componen, además de las diez Asociaciones de Comerciantes existentes en Bilbao, descritas más arriba, El Corte Inglés, la Cámara de Comercio (Sede Oficial) y el Ayun- 
tamiento de Bilbao, este último a través del Área de Salud, Consumo y Comercio (Secretaría de la Asociación) y dos Organismos Autónomos Municipales: Bilbao Iniciativas Turísticas (BIT), cuya Directora Elvira Etxebarría ostenta la Presidencia de Bilbao Dendak, y Lanekintza, S. A., agencia de desarrollo local dependiente de Área Municipal de Empleo.

\section{Plan de actuación 2001}

- Contratación de la GERENCIA DE CENTRO URBANO. En el mes de enero se ha publicado la convocatoria y se espera firmar el convenio de financiación con el Gobierno Vasco a lo largo del mes de febrero. El nombramiento se producirá en el mes de marzo.

- En el mes de enero se ha procedido al registro de la marca «BILBAO DENDAK» y a la adquisición del correspondiente dominio en Internet.

- A lo largo del mes de febrero se presentará el proyecto a potenciales empresas colaboradoras, tales como entidades financieras, socios tecnológicos, etc.

- Puesta en marcha del FORO BILBAO DENDAK.

\section{Foro Bilbao Dendak. Universidad del País Vasco}

\section{Introducción}

El peso específico del sector de la distribución comercial en la economía vasca es muy importante (representa el 12\% del PIB de la CAPV). Sólo en el ámbito de nuestra ciudad, el comercio urbano representa el $30 \%$ de la actividad económica, aglutina miles de empresas y genera más de 20.000 puestos de trabajo.

En la creación de la Asociación para la revitalización del sector comercial de Bilbao, BILBAO DENDAK, y a la hora de programar sus objetivos fundacionales, se destaca como uno de los pilares básicos la colaboración de esta nueva entidad con la Universidad del País Vasco.

Entendemos que en la Universidad, donde ya se vienen tratando y estudiando temas de comercio, se podría desarrollar una labor de coordinación con los intereses del sector de distribución comercial, que redundaría en beneficio de ambos colectivos.

\section{Objetivos}

De lo anteriormente expuesto, podemos deducir una serie de objetivos a lograr como resultado de la colaboración entre BILBAO DENDAK y la Universidad del País Vasco:

- Lograr la interrelación del comercio con la Universidad.

- Identificar y coordinar todos los trabajos relacionados con la Distribución Comercial que se lleven a cabo en las diferentes Facultades de la UPV.

- Fomentar la incorporación de temas de Distribución Comercial en cuantas acciones académicas sea posible.

- Facilitar por parte del comercio representado en BILBAO DENDAK el apoyo a la UPV que resulte de mayor utilidad para el desarrollo de la labor docente; apoyo que debe pasar por una serie de aportaciones y sugerencias que transmitan al ámbito universitario las inquietudes y necesidades reales del sector.

- Analizar todos los posibles campos de actuación de futuro.

\section{Plan de actuación}

Las áreas de trabajo sobre las que podría concretarse el plan de actuación serían:

Estudios

- Posible contratación con la UPV de estudios sobre el sector que encargue BILBAO DENDAK, cualquiera de sus asociaciones miembros, o comercios individuales.

- Promover la realización de trabajo relacionados con la Distribución Comercial por parte de estudiantes, profesores, proyectos de investigación, etc.

\section{Formación}

- Promover la homologación del curso de Asesores Técnicos de Comercio patrocinado por el Gobierno Vasco que en la actualidad se imparte en la UPV.

- Desarrollar cursos de formación específica en colaboración con la Universidad dirigidos al sector comercio.

- Inclusión en la Universidad de Verano de temas de Distribución Comercial.

Para el año 2001 se ha propuesto un curso con el título: «Estrategias del sector comercial en las ciudades del siglo XXI».

En dicho curso se impartirán temas como:

- Análisis de hábitos de consumo.

- Sistemas de Información Geográfica Comercial.

- Sistemas de calidad aplicado al comercio.

- Marketing relacional.

- Los Bussiness Improvement District, un nuevo modelo de gestión urbana. 
- Estrategias de ciudad y su incidencia sobre el tejido comercial urbano.

En este curso está previsto la participación como docentes de varios miembros de la Asociación Española de Gerentes de Centro Urbano (AGECU).

\section{Otras áreas de colaboración}

Cualquier iniciativa de colaboración que pueda plantearse. Ejemplos:

- Organización de jornadas, congresos sobre Distribución Comercial.

- Encuentro sobre el Bilbao comercial del siglo xxI.

- Realización de escaparates de Bilbao con los alumnos de la Facultad de Bellas Artes.

\section{Mecánica de trabajo}

Establecer un comité multidisciplinar estable que se reúna con cierta periodicidad para llevar adelante todas estas propuestas.

Estructurar comisiones de trabajo para acometer proyectos concretos.

Con el fin de dotarle de la máxima relevancia y eficacia a este Foro, pueden incorporarse al mismo representantes del Gobierno Vasco, Diputación Foral, así como cualquier otra Institución o empresa que se considere oportuno.

Se propone finalmente que esta colaboración entre BILBAO DENDAK y la Universidad del País Vasco se plasme en la firma de un documento por parte de los representantes de ambas Instituciones. Dicho documento está previsto que se firme la semana del 23 al 29 de abril, durante la celebración de la

\section{Primavera comercial de Bilbao}

El objetivo de esta semana, que se pretende institucionalizar, es convertir al comercio bilbaíno, sobre todo al agrupado en BILBAO DENDAK, en protagonista de la vida ciudadana desde ámbitos tan dispares como el comercial, el social y el cultural.

Se promoverán con tal motivo campañas de promoción comercial y de animación. Se propondrá a las Asociaciones miembros la realización de campañas en sus ámbitos de actuación. En el ámbito socio-cultural se promoverá la recuperación de la figura de los «Juegos Florales», auténticos dinamizadores de la vida cultural bilbaína del primer tercio del siglo XX y en los que tanto gustaba participar a D. Miguel de Unamuno. Contarán con certámenes de pintura, poesía, actuaciones musicales, etc.

Durante esta semana, y además de otros actos sociales, se presentará el

\section{Premio periodístico Bilbao Dendak}

Este premio se convoca en colaboración con la Asociación de la Prensa de Vizcaya, y podrán participar en él cuantos periodistas lo deseen, en base a trabajos publicados en Vizcaya y cuyo contenido gire en torno a Bilbao, al comercio urbano o a cualquier tema relacionado con la vida ciudadana.

\section{Mall Virtual e-shopping Bilbao}

Durante este año se pretende la coordinación de todos los agentes implicados en el proceso, Instituciones, socios tecnológicos, entidades financieras, asociaciones, comercios, etc. Esta coordinación debe de servir para incorporar en un solo proyecto las iniciativas existentes en diversos ámbitos.

\section{Bilbao comercial siglo XXI}

\section{Introducción}

La estructura del comercio urbano es consecuencia de una serie de factores, unos inherentes al propio comercio y otros exógenos pero absolutamente definitorios del «ser» de la ciudad.

La Villa de Bilbao, con sus características geográficas, históricas y socioeconómicas, ha configurado un tipo de comercio que en el umbral y ante un modelo de ciudad que se vislumbra diferente al hasta ahora existente, se exige un profundo análisis y reflexión de todo el sector para adaptarse a los nuevos tiempos. 
Nuestro objetivo es sentar unas bases sobre las que los distintos colectivos implicados (comercio, administraciones y consumidores) podamos establecer el Bilbao comercial del siglo XXI.

\section{Reflexiones sobre Bilbao}

La configuración de Bilbao define el nivel y el tipo de comercio de la ciudad. Por ello, es necesario analizar su presente y sobre todo sus planes de futuro para poder predecir las líneas de actuación del sector.

\subsection{Modelo de ciudad}

La ciudad está en un proceso de cambio sobre el que habría que definir y consensuar sus grandes líneas, con el fin de que las mismas puedan llevarse a cabo con independencia de situaciones coyunturales.

En este sentido está por definir, al menos de forma definitiva, el carácter de Bilbao como ciudad: industrial, de servicios, turística, burguesa, ..., asumida por todos los ciudadanos y consecuentemente puesta en valor.

\subsection{Plan Estratégico de Bilbao}

La definición del modelo de ciudad debe venir dada por la puesta en marcha de un Plan Estratégico global para la ciudad, que contemple al comercio como uno de los motores fundamentales del desarrollo urbano, el principal foco de visitantes a la ciudad, así como un elemento que aporta calidad social a los barrios.

\section{Puntos fuertes}

- Buen nivel socioeconómico.

- Amplia red de comunicaciones.

- Actitud positiva de las Administraciones.

- Ciudad renovada con resonancia internacional consecuencia de un gran proyecto urbano, que debe ir de la mano de un plan comercial integral.

- Ciudad segura.

\section{Puntos débiles}

- Inexistencia de un plan integral coordinador de intereses comerciales, urbanísticos y de movilidad ciudadana que sirva de guía en las actuaciones individuales que se lleven a cabo.
- Falta de un Plan de Ocio que contemple actividades juveniles, deportivas, infantiles, gastronómicas, etc. Ejemplo: inexistencia de un Palacio de Deportes moderno.

- Diseño de un ambicioso Plan de Marketing de ciudad con gran dotación de medios.

- Deficiencias zonales, sobre todo en equipamientos, en las periferias y, en algunos casos, en el centro.

\section{Modelo comercial urbano}

La primera parte del Plan Estratégico de Revitalización Comercial de Bilbao (PERCO), correspondiente al Análisis de la Demanda, define Bilbao en los siguientes términos:

- Un foco formidable en un entorno crecientemente absorbente: «De la acogida al intercambio».

- Hábitats comerciales heterogéneos: «Los Bilbaos comerciales como transfondo».

- La transformación urbana como respuesta: «Del reto comercial al reto urbano como polo terciario y de calidad de vida».

Si a esto le unimos que de los 256.150 millones de pesetas en que se estima el potencial comercial de Bilbao, el $25 \%$ procede de clientes de fuera de la Villa, podemos deducir que el nivel comercial de Bilbao es muy superior al de las ciudades de su entorno, especialmente desde la incorporación en los últimos dos años de las primeras firmas internacionales y la remodelación de muchos de los establecimientos tradicionales.

El mantenimiento y mejora de este status debe ser uno de los objetivos de los representantes institucionales y del sector, logrando mantener un equilibrio entre los intereses empresariales de los comerciantes y el interés público de la ciudad.

En cualquier caso, es conveniente considerar los siguientes puntos:

- Posicionarse como sector estratégico de la ciudad.

- Transmitir el atractivo comercial de Bilbao en todo el Eje Atlántico.

- Definir una imagen de calidad, modernidad e internacionalidad.

- Potenciar la capacidad de asociarse y liderar proyectos en común.

En definitiva, hay que impulsar, controlar y coordinar su evolución global y zonal.

\section{Plan de actuación comercial}

Bilbao Dendak es el marco en el que se han de identificar los problemas y definir la respuesta del sector comercial de Bilbao. 


\section{Conclusiones}

Todo lo anteriormente expuesto, implica un proceso de estudio de la situación actual y de la deseable, para lo que consideramos necesaria la realización de unas jornadas de tra-

\section{Documentación gráfica}
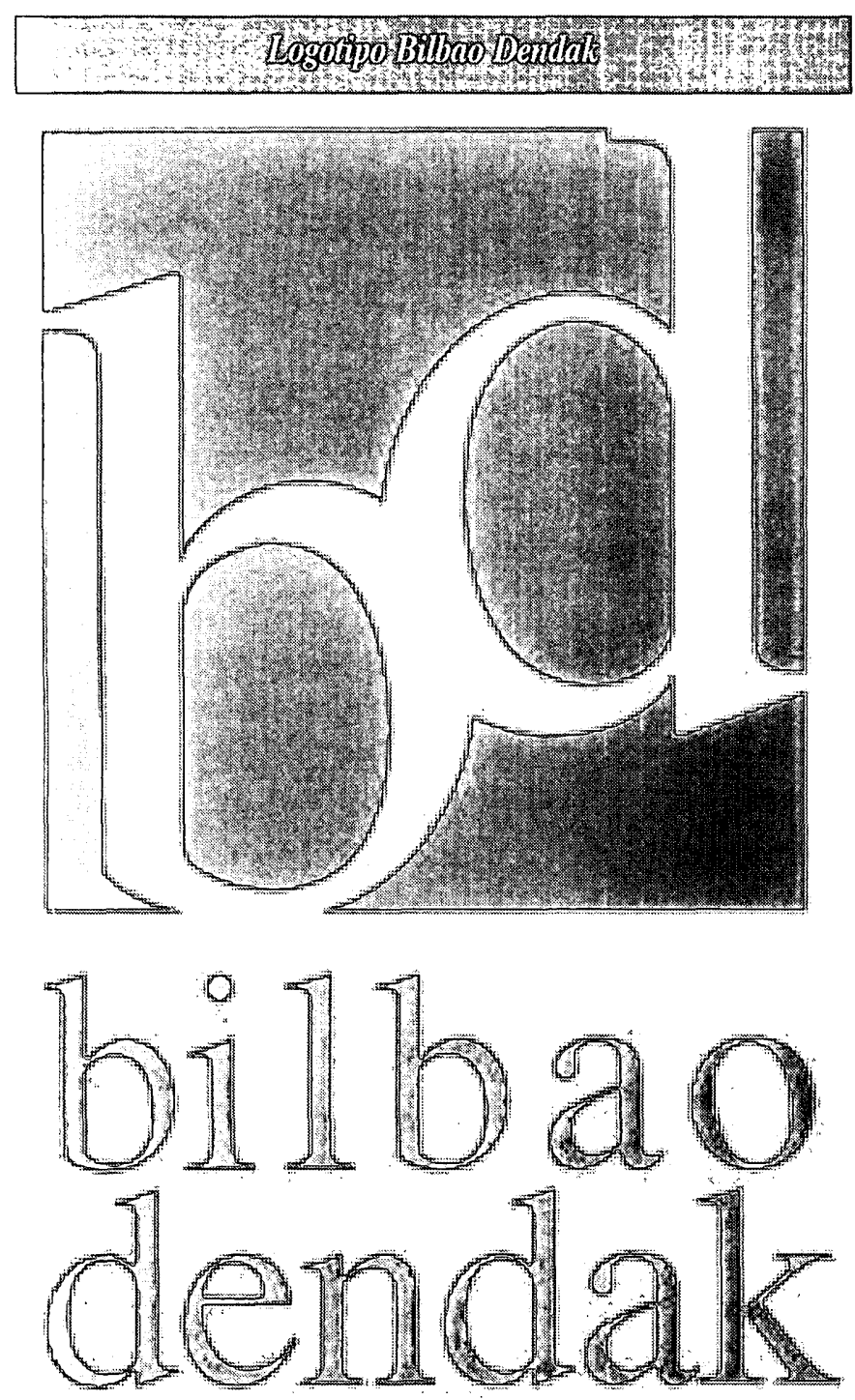

bajo en Bilbao en las que se pongan de manifiesto las posturas de los diferentes agentes urbanos, se expongan experiencias de otras ciudades europeas, etc., de forma que se sienten las bases de un modelo de ciudad comercial para el Bilbao del siglo XXI.

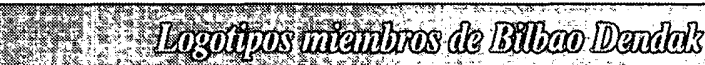

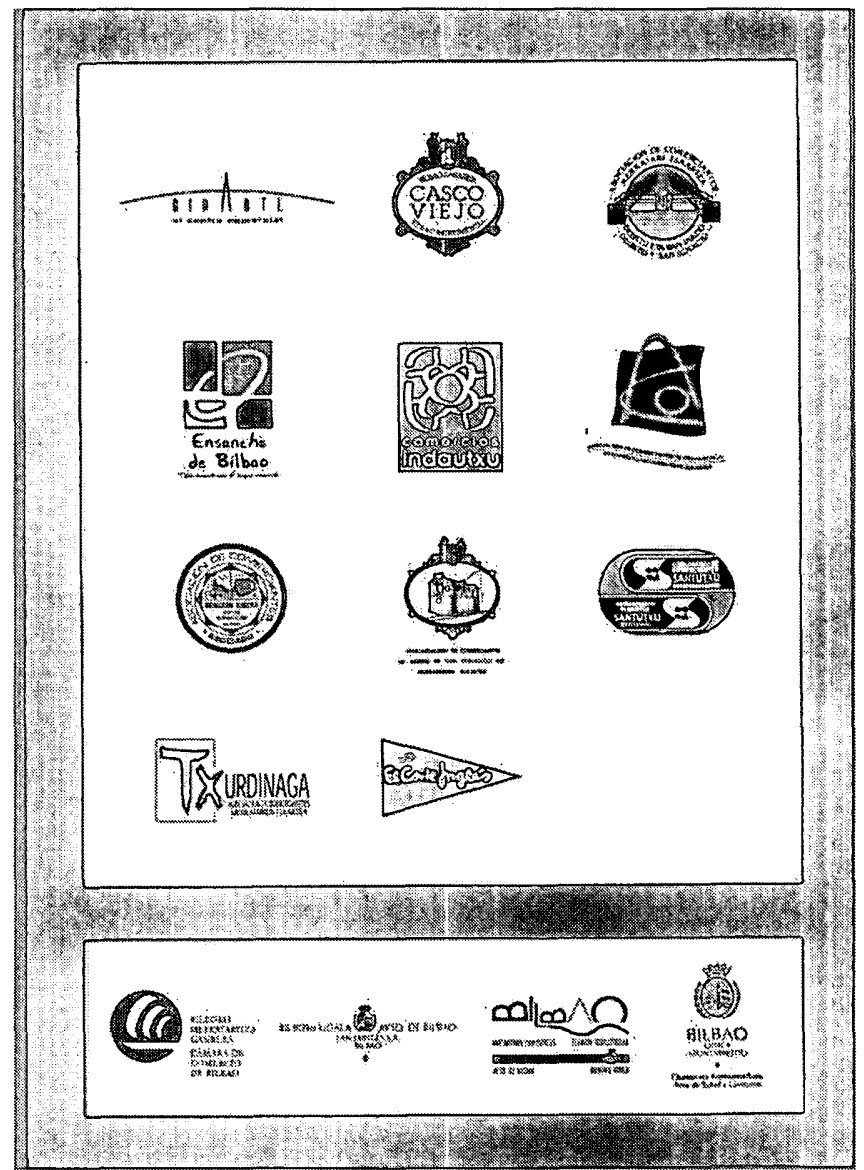




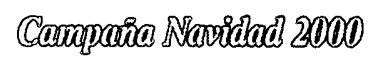

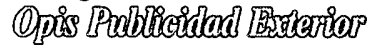

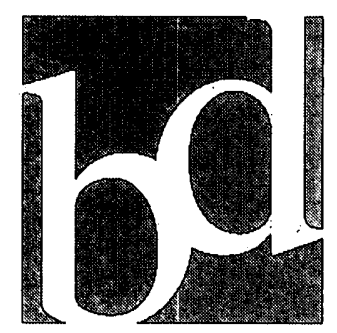

bilbao

dendak

Para ir de compras. Bilbao

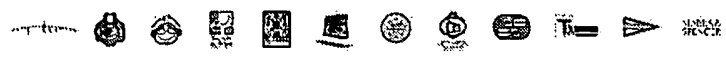

$$
\text { O. }-1-\underline{10}
$$

Canpaña NCarddad 2000 Bublicidad prenso aserith

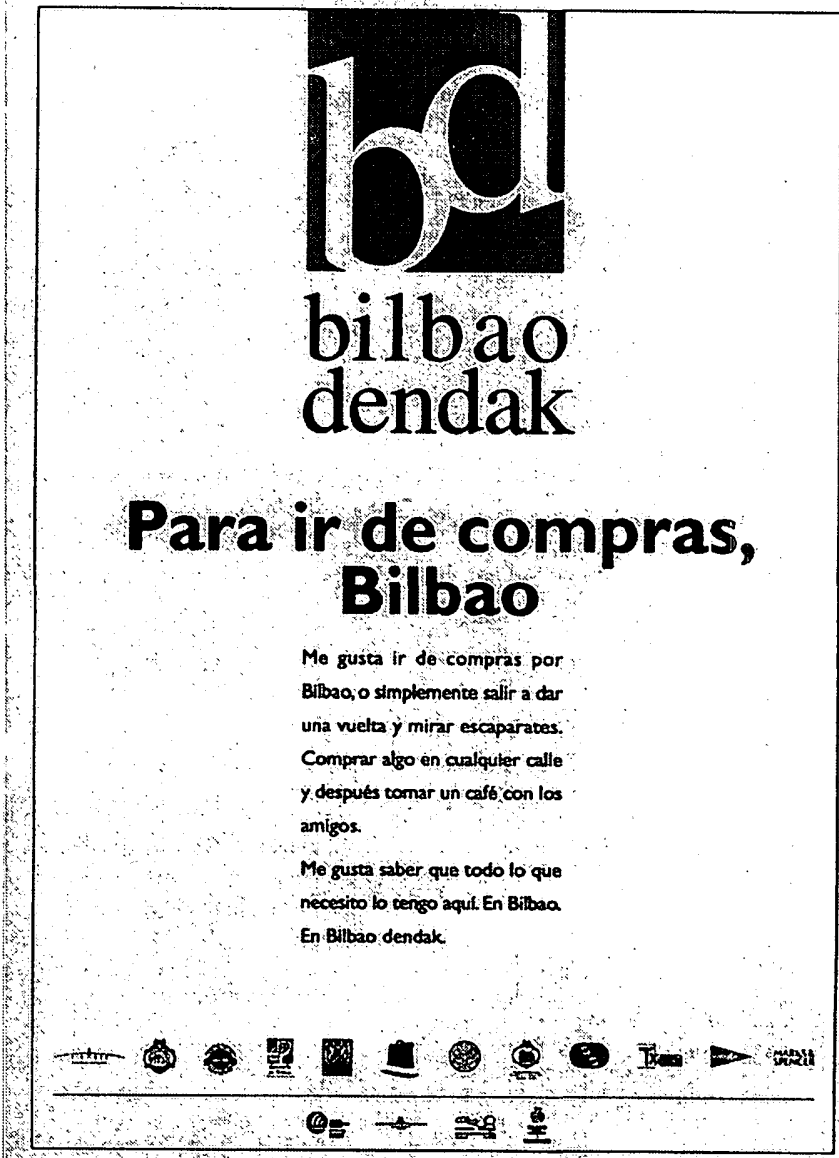

Notas

* Director Gerente de la Asociación de Comerciantes del Casco Viejo de Bilbao. 
